\title{
Evaluating the Catalytic Contribution from the Oxyanion Hole in Ketosteroid Isomerase
}

\author{
Jason P. Schwans ${ }^{\dagger}$, Fanny Sunden ${ }^{\dagger}$, Ana Gonzalez $\$$, Yingssu Tsai ${ }^{\S},+$, and Daniel \\ Herschlag ${ }^{\dagger, \S,+, *}$ \\ tDepartment of Biochemistry, Stanford University, Stanford, CA 94305 \\ +Department of Chemistry, Stanford University, Stanford, CA 94305 \\ $\S$ Stanford Synchrotron Radiation Lightsource, SLAC National Accelerator Laboratory, Menlo Park, \\ CA, 94025
}

\begin{abstract}
Prior site-directed mutagenesis studies in bacterial ketosteroid isomerase (KSI) reported that substitution of both oxyanion hole hydrogen bond donors give a $10^{5}-10^{8}$ fold rate reduction suggesting that the oxyanion hole may provide the major contribution to KSI catalysis. But these seemingly conservative mutations replaced the oxyanion hole hydrogen bond donors with hydrophobic side chains that could lead to suboptimal solvation of the incipient oxyanion in the mutants, thereby potentially exaggerating the apparent energetic benefit of the hydrogen bonds relative to water-mediated hydrogen bonds in solution. We determined the functional and structural consequences of substituting the oxyanion hole hydrogen bond donors and several residues surrounding the oxyanion hole with smaller residues in an attempt to create a local site that would provide interactions more analogous to those in aqueous solution. These more drastic mutations created an active site cavity estimated to be $\sim 650 \AA^{3}$ and sufficient for occupancy by $15-17$ water molecules and led to a rate decrease of only $\sim 10^{3}$-fold for KSI from two different species, a much smaller effect than that observed from more traditional conservative mutations. The results underscore the strong context dependence of hydrogen bond energetics and suggest that the oxyanion hole provides an important, but moderate, catalytic contribution relative to the interactions in the corresponding solution reaction.
\end{abstract}

Understanding the mechanisms that enzymes use to accelerate chemical reactions has been a central goal of Biochemistry. The now-standard tools of site-directed mutagenesis and x-ray crystallography have contributed enormously to our understanding of enzyme action by identifying residues important for catalysis and placing them within the context of the active site. Nevertheless, quantitative understanding of the mechanisms by which enzymes achieve their tremendous rate enhancement remains limited.

Hydrogen bonding to groups that develop charge in the course of a reaction is a prevalent feature of enzyme active sites, and the classic example is stabilization of the incipient oxyanion in the oxyanion hole of serine proteases. ${ }^{1-4}$ Nevertheless, experimental studies of the catalytic contribution from oxyanion hole hydrogen bonds are limited. For most serine proteases the oxyanion hole hydrogen bonds are mediated by backbone amides and therefore cannot be readily mutated. Wells and coworkers used subtilisin in their classic site-directed

*herschla@stanford.edu.

Supporting Information Available. Experimental methods, Figures S1-S7, and Tables S1-S6. This material is available free of charge via the Internet at http://pubs.acs.org. 
mutagenesis studies as one of the oxyanion hole hydrogen bond donors is an Asn side chain, but the other oxyan-ion hole hydrogen bond donor, a backbone amide, and the interplay between the two donors could not be investigated. ${ }^{3,4}$ Further, it is tempting to interpret rate effects from simple mutations as reporting on the contribution of individual residues to catalysis. However, different mutations can give different effects and minimal or 'conservative' mutations may introduce unfavorable effects beyond removal of a functional group, thereby exaggerating the apparent energetic benefit of the enzymatic group. ${ }^{5-7}$

Bacterial ketosteroid isomerase (KSI) has provided a powerful system to investigate the physical and energetic properties of enzymatic hydrogen bonds. ${ }^{7-11}$ KSI catalyzes the double bond isomerization of steroid substrates that involves formation of a dienolate intermediate within an active site oxyanion hole composed of the side chains of Tyr16 and Asp103 and thereby allowing both oxyanion hole hydrogen bond donors to be probed by mutagenesis (Scheme 1). ${ }^{12,13}$ We report herein the effect of fully removing the oxyanion hole hydrogen bond donors and also surrounding residues in an effort to replace the oxyanion hole with an aqueous-like environment. The results suggest a surprisingly small contribution of the oxyanion hole, relative to interactions in the corresponding solution reaction.

Previous studies reported that the Pseudomonas putida (pKSI) and Comamonas testosteroni (tKSI) double mutations that remove the Tyr and Asp hydrogen bond donors in the oxyanion hole result in a $10^{5}$ and $10^{8}$-fold decrease in activity, respectively (pKSI: Y16F/D103L; tKSI Y16F/D103A; pKSI numbering is used throughout for simplicity). ${ }^{14,15}$ The large rate reductions observed in the mutagenesis experiments and additional spectroscopic and computational studies have led to the suggestion that the oxyanion hole provides a major contribution to KSI catalysis. ${ }^{16-18}$ Nevertheless, the conservative mutations replace the oxyanion hole hydrogen bond donors with bulky, hydrophobic side chains that could lead to suboptimal solvation of the incipient oxyanion in the mutants, thereby potentially exaggerating the energetic benefit of the hydrogen bond compared to water-mediated hydrogen bonds in solution. 7,8

Indeed, Kraut et al. reported that whereas the conservative Y16F mutation alone results in a $\sim 10^{5}$-fold effect, mutation of Y16 to the less conservative Ala, Gly, Ser, Thr leads to a much smaller rate reduction of only $\sim 10^{2}$-fold (Figure 1A). ${ }^{7}$ The Y16F mutation was suggested to replace Y16 with a hydrophobic environment, whereas mutation of Y16 to smaller residues appears to replace Y16 with a water-filled cavity that provides aqueous-like solvation of the oxyanion. ${ }^{7}$ These results suggested that the catalytic contribution from the oxyan-ion hole relative to the nonenzymatic solution reaction might be smaller than previously ascribed from the results of seemingly conservative site-directed mutagenesis. ${ }^{14,15}$

Prior studies reported that mutations of the D103 oxyanion hole hydrogen bond donor lead to a rate decrease of $\sim 10^{2}$ and $\sim 10^{3}$-fold for pKSI and tKSI, respectively (pKSI: D103L; tKSI D103A). ${ }^{14,15}$ These mutations again might not create sufficient space for water to replace the enzymatic hydrogen bond donor and could similarly exaggerate the energetic benefit of the enzymatic hydrogen bond donor compared to a water-mediated hydrogen bond.

We set out to determine whether seemingly less conservative oxyanion hole mutations would be less disruptive to KSI catalysis, presumably because hydrogen bonds to water could be restored, and we further tested this model by mutating several residues surrounding the oxyanion hole to potentially create a local site that would provide interactions more analogous to those in aqueous solution. 
Our results with the substrate 5(10)estrene-3,17-dione [5(10)EST] for the pKSI D103A and D103L mutations are similar to the previously reported $\sim 200$-fold rate decrease for the pKSI D103L mutation using the substrate 5-androstene-3,17-dione (5-AND) (Figure 1B and Table S1). ${ }^{14}$ We primarily used 5(10)EST to ensure that a chemical step is rate limiting in all cases and that $K_{\mathrm{M}}$ reflects binding. ${ }^{19}$ Nevertheless, similar results for oxyanion hole mutations were obtained using the steroid substrate, 5-AND, and a one-ring substrate, 3-cyclohexen-1one (Supporting Information and see below). In contrast to the results with D103A and D103L, mutation to a smaller Gly residue at this position gave only a 28 -fold rate decrease (Figure 1B), suggesting that the smaller Gly might open more space and reduce or eliminate unfavorable interactions introduced from the seemingly conservative mutations. If so, there would be a smaller catalytic contribution from the oxyanion hole than previously described from site-directed mutagenesis using conservative mutations.

We next considered both oxyanion hole hydrogen bond donors together. We mutated both pKSI oxyanion hole hydrogen bond donors to either Ala or Gly. To determine if the double mutations introduce structural rearrangements we solved the pKSI Y16A/D103A crystal structure. The overall pKSI Y16A/D103A structure (PDB ID 3T8N) was the same with that of previously determined wild type pKSI (PDB ID 1OPY), with a root-mean-square deviation of $0.3 \AA$ for the backbone atoms and no indication of structural rearrangements (Figure S1). With 5(10)-EST, the Y16A/D103A double mutation led to a 45,000 fold decrease in activity, similar to the $\sim 10^{5}$-fold effect previously reported for Y16F/D103L using 5-AND (Figure 1C). ${ }^{14}$ However, the more drastic Y16G/D103G double mutation decreased activity less, by only 1,200-fold (Figure 1C, red bar, and Figure S2). The $K_{\mathrm{M}}$ values for the oxyanion hole mutants were within two-fold of that for wild-type KSI suggesting that the mutations do not affect ground state binding and indicating near identical rate effects on $k_{\text {cat }}$ and $k_{\text {cat }} / K_{\mathrm{M}}$ (Table S1).

The $~ 40$-fold smaller decrease in activity for the double glycine mutant compared to the double alanine mutant is not the result typically expected for more extensive and less conservative mutation, but the result is consistent with the model that opening more space creates a water-filled cavity that replaces the enzymatic oxyanion hole hydrogen bond donors. Thus, the $\sim 10^{3}$-fold rate reduction for KSI without enzymatic oxyanion hole hydrogen bond donors suggests that the oxyanion hole may provide a significantly smaller catalytic contribution than the $10^{5}-10^{8}$ fold previously observed in site-directed mutagenesis experiments. ${ }^{15}$ Alternatively, the double Gly mutation could introduce fortuitous interactions, possibly from ordered water molecules that mitigate some of the deleterious effect from ablating the enzymatic hydrogen-bonding residues. To further test these models we performed additional functional tests in pKSI and functional and structural tests in the related tKSI.

If the Y16G/D103G double mutant were to render the oxyanion hole effectively water-like, additional mutations that open space would be expected to have little effect on activity. In contrast, if structured water molecules stabilize the incipient oxyanion in the Y16G/D103G double mutant then additional mutations that open more adjoining space would be expected to disrupt the positioned waters, thereby decreasing activity. The extensive mutagenesis necessary to 'excavate' the oxyan-ion hole is likely to disrupt folding or positioning within the structured protein for many enzymes, so that there could be multiple origins of additional deleterious effects. Fortunately, as described below, KSI is amenable to the extensive mutage-nesis necessary to further carve out the oxyanion hole, allowing an in-depth investigation of oxyanion hole catalysis.

The pKSI oxyanion hole hydrogen bond donors (red) are surrounded by two hydrogen bonding residues, Tyr57 and Tyr32 (blue), and a constellation of hydrophobic residues 
(black and green) (Figure 2A). ${ }^{12}$ We mutated the active site tyrosine residues (Figure 2A, blue residues) and multiple surrounding hydrophobic residues (Figure 2A, green residues) to create space. Tests to ensure the stability of these mutants are described in the Supporting Information. Remarkably, the additional mutations led to little change in activity (Figure 2B, blue and green bars) compared to the Y16G/D103G double mutant (Figure 2B, red bar). The similar activity for mutants with additional surrounding groups removed supports the model that mutation of Tyr16 and Asp103 to smaller Gly residue replaces the oxyanion hole with an effectively solution-like site.

We next tested if mutation of the full oxyanion hole and neighboring residues leads to similar results in the related tKSI. The homologous pKSI and tKSI enzymes have the same oxyanion hole hydrogen bonding residues, but the surrounding residues are different so it is unlikely to have the same coincidental effects from mutations that might reposition groups or give pre-positioned water molecules within the oxyanion hole.

Previous studies using the 5-AND steroid substrate reported an enormous rate reduction of $10^{8}$-fold for the tKSI Y16F/D103A double mutation. ${ }^{15}$ With the 5(10)EST steroid substrate, we determined that mutation of both tKSI oxyanion hole hydrogen bond donors to Ala led to a $10^{5}$-fold decrease in activity (Figure S3). Mutation of both oxyanion hole hydrogen bond donors to Gly again led to a still less severe effect of $\sim 10^{4}$-fold using the 5(10)EST and 5AND steroid substrates (Figure S3 \& S4). Mutation of the neighboring Tyr57 to Phe and Ala increased or led to little further change in the oxyan-ion hole background (Figures S3-S5). The similar effects of $\sim 10^{3}$-fold in pKSI and tKSI from mutations to create space in the oxyanion hole (Tables S1-S5) suggest that the smaller rate effects are not from coincidental effects introduced by the mutations.

The functional tests described above assume that the mutations open space for water and that there are no structural rearrangements that enhance function. We were unable to obtain diffractable crystals of the Gly oxyanion hole mutants, but we were able to solve the crystal structure of tKSI Y16A/Y57F/D103A (PDB ID 3T8U). X-ray data and refinement statistics are listed in Table S6. The overall structure obtained at $2.5 \AA$ resolution is nearly identical to that of wild type tKSI (PDB ID 8CHO; $2.3 \AA$ ) ${ }^{20}$, with a root-mean-square deviation of $0.4 \AA$ for backbone atoms. The electron density map for the tKSI Y16A/Y57F/D103A structure, contoured at $1.0 \sigma$, shows well-defined density for the modeled atomic positions of the residues surrounding the oxyanion hole (Figure 3A), with modeled side chain positions nearly superimposable with wild type. Thus, the $\mathrm{x}$-ray crystallographic results suggest the mutations do not induce structural rearrangements (Figure S6). Removal of the oxyanion hole residues generates a cavity of approximately $150 \AA^{3}$ (Figure 3B), sufficient to accommodate four to five waters. ${ }^{21}$ The subsequent mutations of surrounding groups in tKSI and pKSI presumably allow access of additional water molecules with space sufficient to accommodate $15-17$ waters in the most severe mutants. ${ }^{21}$ As activity is increased for multiple variants relative to the Ala oxyanion hole mutants, these mutations presumably do not cause deleterious active site rearrangements.

The results herein indicate that mutations that ablate both oxyanion hole hydrogen bond donors and open space for water result in an $\sim 10^{3}$ to $\sim 10^{5}$-fold smaller rate decrease in pKSI and tKSI compared to the more conservative $\mathrm{Y} 16 \mathrm{~A} / \mathrm{D} 103 \mathrm{~A}$ described herein and the $\mathrm{Y} 16 \mathrm{~F} /$ D103A mutation previously reported. ${ }^{15}$ The variation in rate effects with different mutations underscores that hydrogen bond energetics and mutage-nesis experiments are highly context dependent. In the nonen-zymatic solution reaction water can rearrange to solvate the incipient oxyanion (Figure 4A). Wild-type KSI has two oxyanion hole hydrogen bond donors positioned to hydrogen bond with the oxyanion (Figure 4B). The 'conservative' mutations are suggested to replace the enzymatic hydrogen bond donors with a hydrophobic 
environment that desolvates the localized negative charge in the transition state relative to aqueous solution (Figure 4C, gray shading), whereas water in the carved-out mutant behaves in a more solution-like manner, arranging to stabilize the localized negative charge (Figure $4 \mathrm{D}$, blue shading). In principle, the rate reduction with the carved out oxyanion hole could be lessened by formation of three hydrogen bonds from water molecules in the newly introduced aqueous-like environment rather than two hydrogen bonds in the wild-type KSI active site (Figure $4 \mathrm{~A}$ and $\mathrm{B}$ ).

These and other aspects of the energetics of hydrogen bonding are complex. Hydrogen bonds donated by the enzyme are expected to be energetically favorable relative to hydrogen bonds donated by water as the enzymatic groups have lower $\mathrm{p} K_{\mathrm{a}}$ values than water and are thus stronger hydrogen bond donors. ${ }^{22,23}$ In addition, the enzymatic hydrogen bonding groups are prepositioned to favor hydrogen bonding in the transition state, whereas water molecules must rearrange to optimize interactions in the transition state. ${ }^{8,24}$ Hydrogen bond energetics are also sensitive to the environment that surrounds the hydrogen bonds that are formed, and a favorable process that involves charge accumulation in aqueous solution can be unfavorable in other environments. 8,25

Enzymes catalyze reactions relative to the nonenzymatic reaction in water. Replacing the oxyanion hole hydrogen bond donors with a water-filled cavity provides a crude estimate of the catalytic contribution from the oxyanion hole hydrogen bonds compared to the analogous reaction in water in which there are water-mediated hydrogen bonds. Figure 5 compares the second-order KSI reaction with the second-order acetate-catalyzed reaction, where acetate provides the same general acid/base moiety as the enzyme active site. Prior results have indicated that wild-type KSI provides a $10^{11}-10^{12}$ fold rate increase above the acetate-catalyzed isomerization of 5(10)EST..$^{8,26,27} \mathrm{KSI}$ with the oxyanion hole and surrounding residues removed provides an $\sim 10^{9}$-fold rate increase compared to the acetatecatalyzed reaction, only $\sim 10^{3}$-fold less than wild-type KSI. These comparisons strongly suggest that the oxyanion hole provides an important, but moderate, catalytic contribution and focus us on the task of uncovering how binding interactions and general base catalysis can provide the remaining substantial contribution to overall KSI catalysis. ${ }^{28}$

The $\sim 10^{3}$-fold rate reduction for full ablation of the KSI oxyanion hole is similar to the rate effects previously reported for mutation of the Asn oxyanion hole hydrogen bond donor to Ala, Leu, Thr, and Gln in subtilisin, a serine protease. ${ }^{3,4}$ While the effect from ablating all oxyanion hole hydrogen-bonding groups in subtilisin could be larger than the effects reported for single mutations, it is also possible that the oxyanion hole is no longer utilized in the mutants such that the overall modest effect of the oxyanion hole is general. Support for the latter model arises from the observation of incipient oxyanions pointed away from serine protease oxyanion holes ${ }^{29,30}$ and the similar effect from mutation of the oxyanion hole Asn of sub-tilisin and Kex2 to neutral residues and to the presumably negatively charged Asp residue. ${ }^{4,31}$ It will be fascinating to ascertain the generality versus idiosyncrasies of oxyanion hole and other catalytic contributions between different enzymes by using extensive mutagenesis akin to that carried out herein.

\section{Supplementary Material}

Refer to Web version on PubMed Central for supplementary material.

\section{Acknowledgments}

We thank members of the Herschlag for comments on the manuscript. This work was funded by a National Science Foundation grant MCB-1121778 (to D.H.). J.P.S. was supported in part by a National Institutes of Health (NIH) Postdoctoral Fellowship. Portions of this research were carried out at the Stanford Synchrotron Radiation 
Laboratory, a national user facility operated by Stanford University on behalf of the U.S. Department of Energy, Office of Basic Energy Sciences. The SSRL Structural Molecular Biology Program is supported by the Department of Energy, Office of Biological and Environmental Research, and by the NIH, National Center for Research Resources (NCRR), Biomedical Technology Program, and the National Institute of General Medical Sciences. The project described was partially supported by Grant Number 5 P41 RR001209 from the NCRR, a component of the NIH.

\section{References}

1. Kraut J. Annu Rev Biochem. 1977; 46:331-358. [PubMed: 332063]

2. Robertus JD, Kraut J, Alden RA, Birktoft JJ. Biochemistry. 1972; 11:4293-4303. [PubMed: 5079900]

3. Bryan P, Pantoliano MW, Quill SG, Hsiao HY, Poulos T. Proc Natl Acad Sci USA. 1986; 83:37433745. [PubMed: 3520553]

4. Wells JA, Cunningham BC, Graycar TP, Estell DA. Philos Trans R Soc Lond A. 1986; 317:415423.

5. Kraut DA, Carroll KS, Herschlag D. Annu Rev Biochem. 2003; 72:517-571. [PubMed: 12704087]

6. Fersht, AR. Structure and Mechanism in Protein Science. 2. W.H. Freeman and Company; New York: 1999.

7. Kraut DA, Sigala PA, Fenn TD, Herschlag D. Proc Natl Acad Sci USA. 2010; 107:1960-1965. [PubMed: 20080683]

8. Kraut DA, Sigala PA, Pybus B, Liu CW, Ringe D, Petsko GA, Herschlag D. PLoS Biol. 2006; 4:501-519.

9. Sigala PA, Caaveiro JM, Ringe D, Petsko GA, Herschlag D. Biochemistry. 2009; 48:6932-6939. [PubMed: 19469568]

10. Sigala PA, Kraut DA, Caaveiro JM, Pybus B, Ruben EA, Ringe D, Petsko GA, Herschlag D. J Am Chem Soc. 2008; 130:13696-13708. [PubMed: 18808119]

11. Zhao Q, Abeygunawardana C, Gittis AG, Mildvan AS. Biochemistry. 1997; 36:14616-14626. [PubMed: 9398180]

12. Ha NC, Choi G, Choi KY, Oh BH. Curr Opin Struct Biol. 2001; 11:674-678. [PubMed: 11751047]

13. Pollack RM. Bioorg Chem. 2004; 32:341-353. [PubMed: 15381400]

14. Choi G, Ha NC, Kim SW, Kim DH, Park S, Oh BH, Choi KY. Biochemistry. 2000; 39:903-909. [PubMed: 10653633]

15. Thornburg LD, Goldfeder YR, Wilde TC, Pollack RM. J Am Chem Soc. 2001; 123:9912-9913. [PubMed: 11583562]

16. Cleland WW, Frey PA, Gerlt JA. J Biol Chem. 1998; 273:25529-25532. [PubMed: 9748211]

17. Zhao Q, Abeygunawardana C, Talalay P, Mildvan AS. Proc Natl Acad Sci USA. 1996; 93:82208224. [PubMed: 8710850]

18. Feierberg I, Aqvist J. Biochemistry. 2002; 41:15728-15735. [PubMed: 12501201]

19. Pollack RM, Bantia S, Bounds PL, Koffman BM. Biochemistry. 1986; 25:1905-1911. [PubMed: 3707917]

20. Cho HS, Choi G, Choi KY, Oh BH. Biochemistry. 1998; 37:8325-8330. [PubMed: 9622484]

21. Tsai J, Taylor R, Chothia C, Gerstein M. J Mol Biol. 1999; 290:253-266. [PubMed: 10388571]

22. Hine J. J Am Chem Soc. 1972; 94:5766-5771.

23. Stahl N, Jencks WP. J Am Chem Soc. 1986; 108:4196-4205.

24. Warshel A. J Biol Chem. 1998; 273:27035-27038. [PubMed: 9765214]

25. Shan SO, Herschlag D. Meth Enzymol. 1999; 308:246-276. [PubMed: 10507008]

26. Schwans JP, Kraut DA, Herschlag D. Proc Natl Acad Sci USA. 2009; 106:14271-14275.

[PubMed: 19706511]

27. Zeng B, Pollack RM. J Am Chem Soc. 1991; 113:3838-3842.

28. Wilde TC, Blotny G, Pollack RM. J Am Chem Soc. 2008; 130:6577-6585. [PubMed: 18426205]

29. Bobofchak KM, Pineda AO, Mathews FS, Di Cera E. J Biol Chem. 2005; 280:25644-25650.

[PubMed: 15890651] 
30. Simon L, Goodman JM. J Org Chem. 2010; 75:1831-1840. [PubMed: 20039621]

31. Brenner C, Bevan A, Fuller RS. Curr Biol. 1993; 3:498-506. [PubMed: 15335687] 


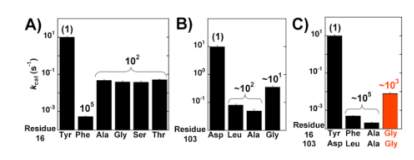

Figure 1.

Effects of oxyanion hole hydrogen bond donor mutations on pKSI activity $\left(k_{\text {cat }}\right)$ using the substrate 5(10)EST. A) Effects of Tyr16 mutations replotted from Kraut et al.; ${ }^{8}$ (B) effects of Asp103 mutations; and (C) effects of full ablation of oxyanion hole hydrogen bond donors. Values are from Table $\mathrm{S} 1$. A comparison of $k_{\text {cat }} / K_{\mathrm{M}}$ values reveals a similar trend (Table S1). 


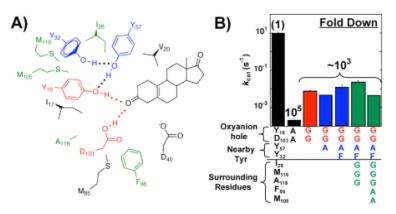

Figure 2.

'Carving out' the pKSI oxyanion hole. A) Schematic representation of oxyanion hole hydrogen bond donors (red), tyro-sine network (blue), and surrounding residues (green and black). The substrate is also shown in black. B) Rate effects from ablating neighboring side chains in the oxyanion hole mutant background. Values are from Table S1. Bars and residues are colored according to panel $\mathrm{A}$. 

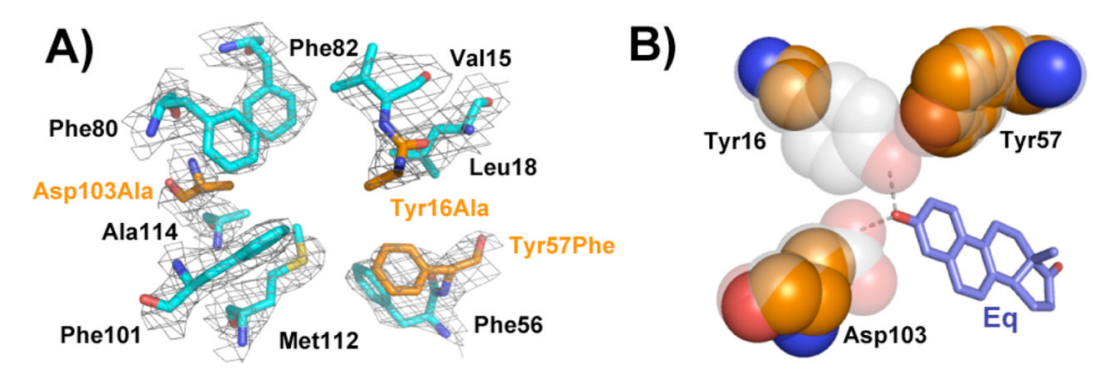

Figure 3.

Crystal structure of tKSI Y16A/Y57F/D103A reveals a cavity in place of the oxyanion hole. A) Sigma-A weighted $2 F_{0}-F_{\text {c }}$ electron density for active site residues (contoured at $1.0 \sigma$ ). Side chains of the mutated residues are colored orange. B) Space filling representation of the oxyanion hole residues. Wild type oxyanion hole hydrogen bond donors and Tyr57 are transparent to show the mutant cavity. 
A)
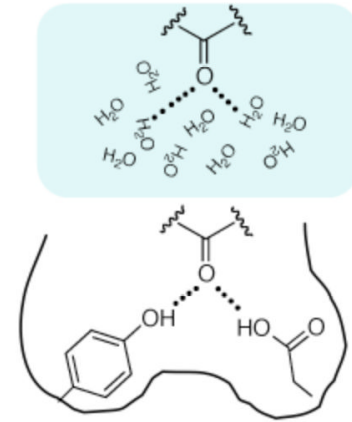

C)

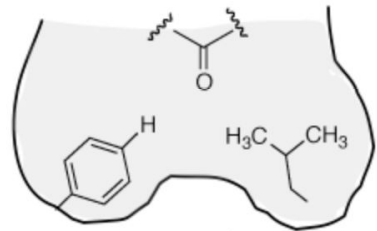

D)

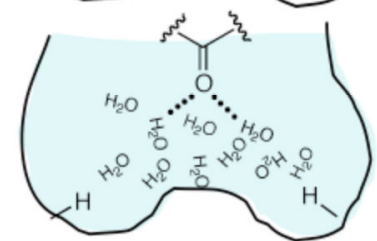

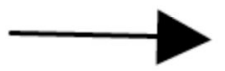
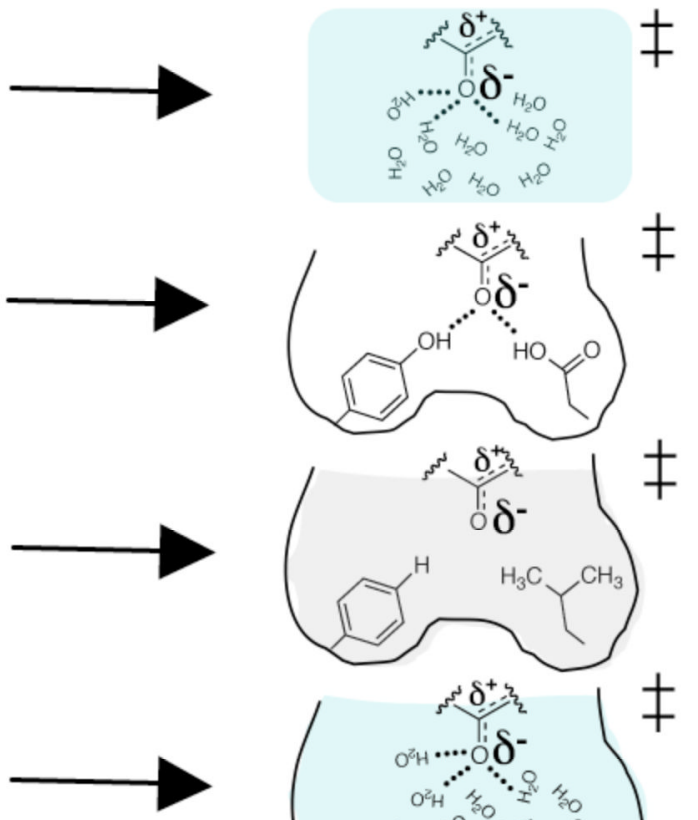

Figure 4.

Schematic models for the effects of oxyanion hole mutations on KSI catalysis. A) Water molecules rearrange in the solution nonenzymatic reaction to provide stabilization of the oxyanion from two or three hydrogen bonds. B) The wild-type KSI oxyanion hole has two positioned hydrogen bond donors that stabilize the incipient oxyanion. C) Conservative mutation of the oxyanion hole hydrogen bond donors ablate enzymatic hydrogen bonds and introduce a neighboring hydrophobic environment. D) Less-conservative, smaller mutations ablate the enzymatic hydrogen bonds but create a cavity that allows water to solvate the incipient oxyanion. Figure adapted from Kraut et al. ${ }^{7}$ 


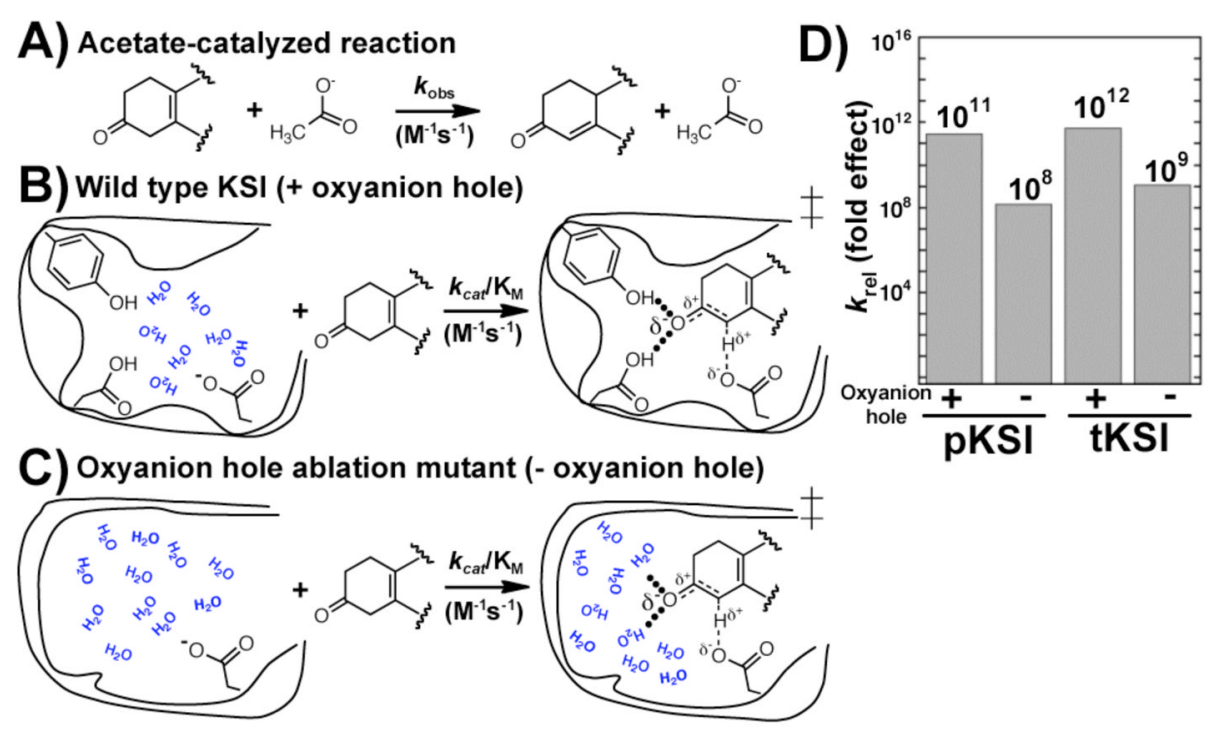

Figure 5.

Determining the catalytic contribution from the oxyan-ion hole in KSI. A) The second-order solution reaction of a KSI substrate with acetate ion. B) The second-order reaction of wild type KSI with the same substrate. C) The second-order reaction of the KSI oxyanion hole ablation mutant. D) Comparison of the second-order rate constant with the second-order nonenzymatic acetate-catalyzed reaction rate constant to measure the overall rate increase for KSI relative to the solution reaction that uses the same general base functionality. Comparison of reaction from the $\mathrm{KSI} \mathrm{E} \bullet \mathrm{S}$ complex relative to the acetate-catalyzed reaction is given in Figure S7. 


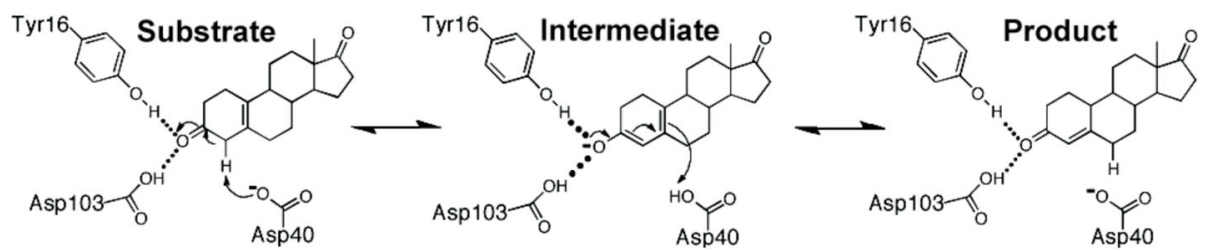

Scheme 1.

Mechanism of KSI-catalyzed isomerization 